\title{
A single-photon emitter using excitation energy transfer between quantum dots
}

\author{
Tadashi Kawazoe, ${ }^{\text {a }}$ Shunsuke Tanaka, ${ }^{a}$ and Motoichi Ohtsu ${ }^{\text {a, b }}$ \\ ${ }^{a}$ Department of Electrical Engineering and Information Systems, Graduate School of \\ Engineering, University of Tokyo, Bunkyo-ku, Tokyo 113-8656, Japan \\ kawazoe@ee.t.u-tokyo.ac.jp \\ ${ }^{\mathrm{b}}$ Japan Science and Technology Agency, Room 213, Bld. 9, University of Tokyo,2-11-16 \\ Yayoi, Bunkyo-ku, Tokyo 113-8656, Japan \\ ohtsu@ee.t.u-tokyo.ac.jp
}

\begin{abstract}
We demonstrated a high-quality single-photon emitter based on excitation energy transfer between two different-sized $\mathrm{CuCl}$ quantum dots (QDs). It is operated under triple blockade mechanisms. The mechanisms are tuning the incident light to the smaller QD and using an electric dipole forbidden excitation energy level in the larger QD, using the optical near-field interaction to transfer energy from the smaller QD to the electric dipole forbidden level of the larger QD, and using a single exciton emission level in the larger QD. These mechanisms are supported by the large binding energy of the exciton molecule. A $99.3 \%$ plausibility of single-photon emission was confirmed with $99.98 \%$ accuracy based on a photon correlation experiment with $80-\mathrm{MHz}$ repetition frequency using an optical fiber probe.
\end{abstract}

Keywords: near-field scanning microscopy and spectroscopy, quantum dots, single-photon emission.

\section{INTRODUCTION}

The single-photon (SP) emitter is one of the key devices required to improve the future performance of optical communication systems and quantum computing [1]. To realize high emission efficiency and suppress the noise due to multiple-photon emission, numerous SP emitters have been developed using quantum dots (QDs) [2-4]. Independently of this work, we have proposed novel nanophotonic devices [5-8] that operate with a single photon and can be used as controllable SP sources. The excitation energy transfer between QDs is the operation mechanism of these devices. Here, we propose a novel SP emitter using near-fieldcoupled $\mathrm{CuCl}$ QDs and demonstrate its operation. Our experimental result provides direct evidence for the SP operation of nanophotonic devices [5-8].

\section{PRINCIPLE AND EXPERIMENTAL SET-UP}

Figure 1(a) shows the proposed SP emitter. It consists of two different-sized $\mathrm{CuCl}$ cubic QDs (QD-A and QD-B) coupled by an optical near-field (ONF) interaction. In $\mathrm{CuCl}$ QDs, the quantized exciton energy level is represented by the three quantum numbers $\left(n_{x}, n_{y}, n_{z}\right)[5,9]$. Assuming the side length $L$ of the $\mathrm{CuCl}$ QDs to be $3.5 \mathrm{~nm}$ and $4.6 \mathrm{~nm}$ (QD-A and QD-B, respectively), the effective size ratio is $1: \sqrt{2}$ when the dead-layer effect is taken into account [9]. As a result, the quantized exciton energy level $\left(n_{x}, n_{y}, n_{z}=1,1,1\right)$ of QD-A resonates with the level $(2,1,1)$ of QD-B.

A SP can be emitted from the exciton energy level $(1,1,1)$ of QD-B by the three blockade mechanisms described below. The resonant excitation by the input light generates a single exciton in the $(1,1,1)$ level of QD-A only because the $(2,1,1)$ level in QD-B is electric dipole forbidden [5]. If we try to generate the second exciton in QD-A [i.e., to generate the exciton 
pair in the $(1,1,1)$ level], its level is redshifted by $30 \mathrm{meV}$, which corresponds to the binding energy of the exciton molecule. This implies that the energy level becomes detuned from the input light, prohibiting exciton generation. Because the separation between the two QDs is less than $30 \mathrm{~nm}$, the exciton in QD-A is transferred to the $(2,1,1)$ level of QD-B by a resonant ONF interaction $[5,10]$ prior to the recombination of the exciton. This transfer occurs despite the electric dipole forbidden feature of the $(2,1,1)$ level. It is also only possible when a single exciton exists in QD-A. This is because the $(1,1,1)$ level of QD-A becomes off-resonant to the $(2,1,1)$ level of QD-B if multiple excitons are generated in QD-A. After the energy transfer, a single exciton can remain stable in QD-B due to the large binding energy of the exciton molecule. Hence, a SP is emitted from the $(1,1,1)$ level of QD-B after fast relaxation from the $(2,1,1)$ to the $(1,1,1)$ level. As a result of these three blockade mechanisms, our proposed SP emitter has a dramatically reduced possibility of multiple-photon emission compared to conventional SP emitters, which are based on only one blockade mechanism that relies on the emission energy shift occurring in a multiple exciton state [3].

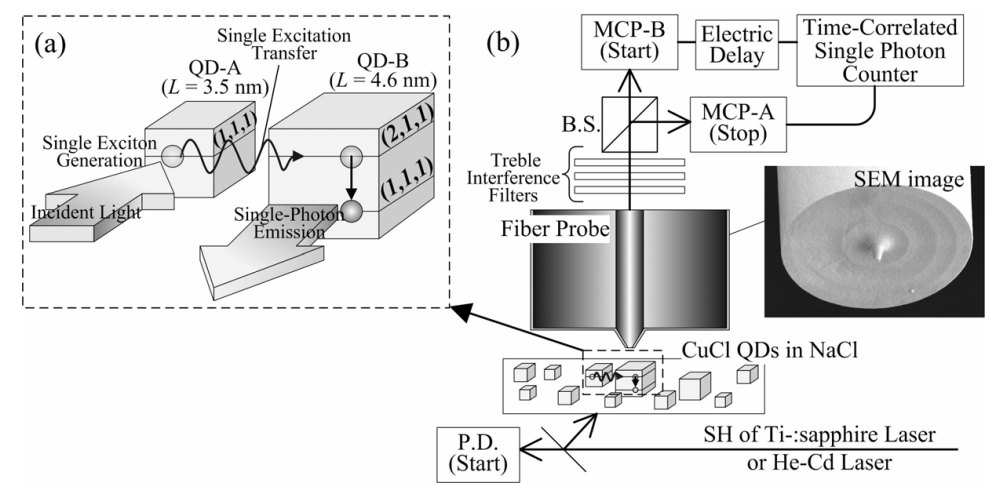

Fig. 1. (a) Configuration of a single-photon emitter. The closely located $\mathrm{CuCl}$ QDs (QD-A and QD-B) had side lengths of $3.5 \mathrm{~nm}$ and $4.6 \mathrm{~nm}$, respectively. (b) Experimental setup to test the single-photon emission using the Hanbury Brown and Twiss method. MCP: Microchannel Plate, B.S.: Beam Splitter, P.D.: Photodiode.

Figure 1(b) shows the setup of the experiment used to test this SP emission mechanism. The $\mathrm{CuCl}$ cubic QDs, with an average side length $L=4.5 \mathrm{~nm}$ (including 3.5-nm QDs and 4.6$\mathrm{nm}$ QDs), were embedded in a $\mathrm{NaCl}$ matrix crystal [11]. In this sample, the electric dipole forbidden feature of the $(2,1,1)$ exciton level was established for the far-field light for the following reasons. (1) The exciton wave function was strongly localized and isolated in the QDs, due to the high potential barrier of $\mathrm{NaCl}$ with a band gap of $7 \mathrm{eV}$. Therefore, the mixing of the exciton states between QDs was negligibly small. (2) The electric field effect due to the intrinsic polarity of QD was also negligibly small, because the blue shift due to the quantum size effect precisely agreed with the quantum confinement effect, without consideration of the polarity of the QDs [9]. The cleaved surface was used for the NFO spectroscopy. The average density of the QDs was $10^{17} / \mathrm{cm}^{3}$ and the average distance between adjacent QDs was less than $30 \mathrm{~nm}$. The proximity of the QDs made possible an energy transfer between adjacent resonant pairs via the ONF interaction $[5,10]$. A CW He-Cd laser $(\lambda=325 \mathrm{~nm})$ was used to find the QD-pair in $\mathrm{NaCl}$ that was acting as a SP emitter. To operate the SP emitter, we used the second harmonic (SH) of a mode-locked Ti:sapphire laser with a pulse duration of 2 ps and a repetition rate of $80 \mathrm{MHz}$. A photodiode (P.D.) was used to monitor the excitation pulse power, and as a trigger source for time-resolved measurements. The sample crystal was excited from its back-surface, under a total reflection condition to prevent the detection of direct laser scattering. The photons emitted from QD-B were collected using an ONF fiber probe with an aperture diameter of $40 \mathrm{~nm}$. The UV optical fiber probe was fabricated using 
chemical etching [12] and coated with a 150-nm-thick Al film. The sample temperature was maintained at $15 \mathrm{~K}$. The Hanbury Brown and Twiss method [13] was used to detect the SP from QD-B. The emitted SP was selected by the treble interference filters, with a center wavelength of $383 \mathrm{~nm}$ and a bandwidth of $1 \mathrm{~nm}$. Use of the total reflection condition and the treble interference filters ensured that the error rate due to the leaked excitation laser pulse was negligible. A microchannel plate (MCP) detector (R3809U-52) was available at the wavelength used. Its quantum efficiency was $25 \%$ at $\lambda=383 \mathrm{~nm}$, and its dark counting rate was 30 counts/s. Because the dark counting rate could be suppressed to below 1 count $/ \mathrm{s}$ by the discriminator in the time-correlated single-photon counter, its contribution to the measurement of the cross-correlation coefficient by synchronous counting was less than $4 \times$ $10^{-10}$ counts/s in the 200-ns observation time window (Fig. 4). As a result, the counting noise due to dark counts was negligible for the accumulation times of 24,000 s and 12,000 s for the measurements shown in Figs. 4(a) and 4(b), respectively.

\section{EXPERIMENTAL RESULTS AND DISCUSSIONS}

Figures 2(a) and (b) show the spatial distributions of the photoluminescence (PL) intensity in identical areas for 383.0-nm and 379.5-nm wavelengths, respectively, using the CW $\mathrm{He}-\mathrm{Cd}$ laser. They were measured using the ONF microscope to find the appropriate QD pair to be used as SP emitters. The He-Cd laser $(\lambda=325 \mathrm{~nm})$ could excite almost all QDs with the size inhomogeneity, because most of the QDs larger than $L=2 \mathrm{~nm}$ had many exciton states resonating at this wavelength, and the number of QDs smaller than $L=2 \mathrm{~nm}$ was negligible in the sample used. Thus, we could observe the PL from most QDs and find the size-selective distribution. The PL wavelengths $(383.0 \mathrm{~nm}$ and $379.5 \mathrm{~nm})$ corresponded to the $(1,1,1)$ levels of 4.6-nm and 3.5-nm QDs, respectively.
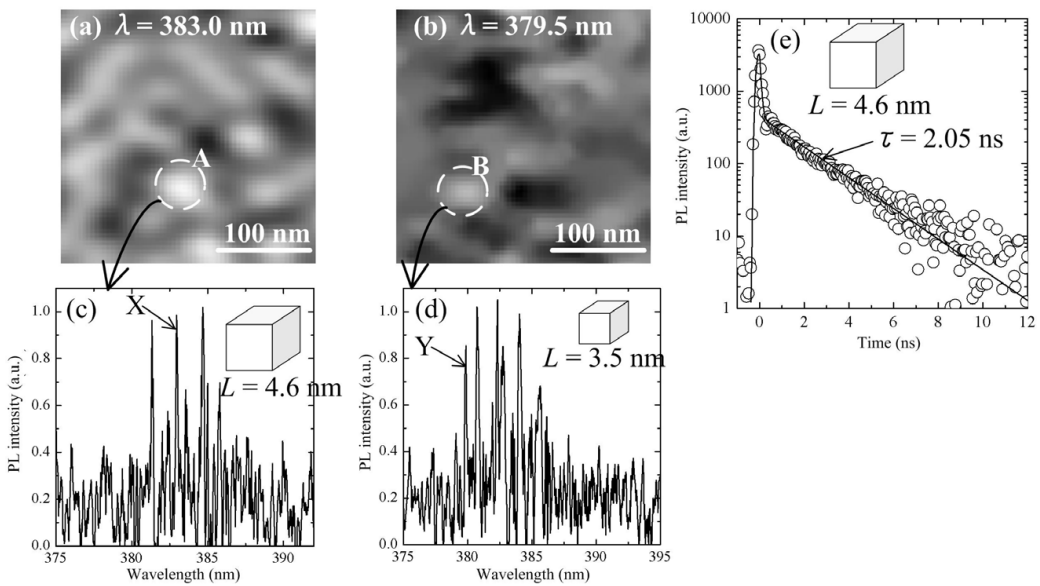

Fig. 2. Spatial distribution of the ONF-PL from (a) 4.6-nm and (b) 3.5-nm QDs. The ONF spectra from (c) region A and (d) region B. (e) Time evolution of PL from the 4.6-nm QD in region A.

Figures 2(c) and (d) show the near-field PL spectra, which were observed in regions A and B surrounded by the dashed open circles [Figs. 2(a) and 2(b), respectively]. Figure 2(c) shows the spectral peak $X$ at $383.0 \mathrm{~nm}$, which was due to the $(1,1,1)$ 4.6-nm QD level. Peaks at $379.5 \mathrm{~nm}$ from the $(1,1,1) 3.5-\mathrm{nm}$ QD level were not observed in that region. Conversely, Fig. 2(d) shows the spectral peak Y at $379.5 \mathrm{~nm}$ from the $(1,1,1) 3.5-\mathrm{nm}$ QD level, but peak X is not present. Because the spectral peaks of Figs. 2(c) and 2(d) have these anti-correlation features, and the distance between regions A and B is only $30 \mathrm{~nm}$, we concluded that a part of 
energy is transferred from the 3.5-nm QD in region $\mathrm{B}$ to the 4.6-nm QD in region $\mathrm{A}$. Therefore, these two regions comprise a QD pair for the SP emitter.

Next, we fixed the fiber probe to region A to test whether SP emission had occurred. Figure 2(e) shows the time evolution of the PL peak X from the 4.6-nm QD when excited by the $\mathrm{SH}$ of the mode-locked Ti:sapphire laser. The excitation power density was $0.1 \mathrm{~W} / \mathrm{cm}^{2}$. From the PL decay time, the exciton lifetime was estimated as 2.05 ns. Therefore, when the SP emitter was driven at a repetition frequency of $80 \mathrm{MHz}$, the probability of error was due to the asynchronous emission of a SP was $0.23 \%[=\exp (-12.5 / 2.05)]$.

Figure 3(a) shows the result of the synchronous counting between the P.D. and MCP-A. Synchronous counting was performed to estimate the efficiency of the SP emission. The wavelength of the SH of the mode-locked Ti:sapphire laser was tuned to the $(1,1,1) 3.5-\mathrm{nm}$ QD level $(\lambda=379.5 \mathrm{~nm})$, to realize the configuration of a SP emitter, as shown in Fig. 1 (a). The excitation power density, $10 \mathrm{~W} / \mathrm{cm}^{2}$, was relatively high. However, the thermal effect due to this high power density appeared negligible because the decay time of each pulsive profile in the curve of Fig. 3(a) was close to the estimated PL decay time (2.05 ns) of the 4.6-nm QD. The thick black bars in Fig. 3(b) show the number of counts integrated over a repetition interval of $12.5 \mathrm{~ns}$. From this figure, we estimated that the observed photon-counting rate for both MCP-A and MCP-B was $1 \times 10^{4}$ counts/s. The observation efficiency (i.e., the external efficiency of the SP emitter) was estimated to be approximately $1.3 \times 10^{-4}$ [ $=$ (the counting rate $) /($ the excitation repetition rate $\left.)=1 \times 10^{4} / 80 \times 10^{6}\right]$. The total extraction efficiency was $1.3 \times 10^{-4}\left(=1 \times 10^{-3} \times 0.5 \times 0.25\right)$. In order, the values in this equation were the approximate throughput efficiency of the fiber probe, the efficiency of the treble interference filters (including the coupling efficiency), and the quantum efficiency of the MCP. Therefore, the internal efficiency of the SP emission was estimated to be $100 \%$ [ $=$ (the external efficiency $) /($ the total extraction efficiency $\left.)=\left(1.3 \times 10^{-4} / 1.3 \times 10^{-4}\right) \times 100\right]$.
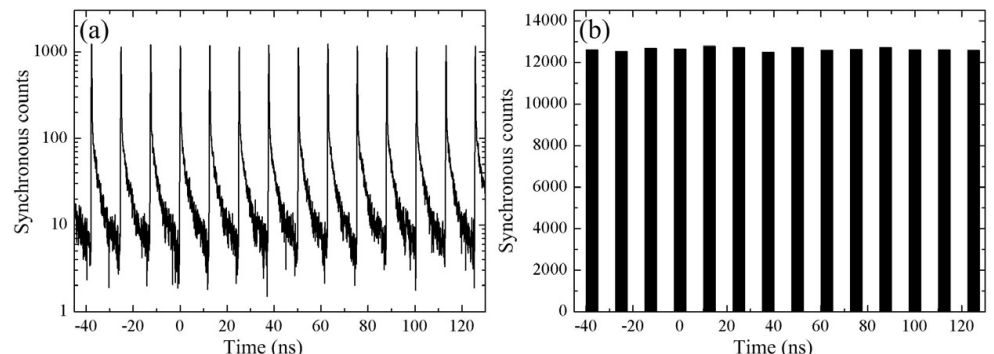

Fig. 3. (a) Synchronous counting between the P.D. and MCP-A used to measure the single-photon generation efficiency. (b) The same counts integrated over $12.5 \mathrm{~ns}$.

The thick black bars in Fig. 4 show the experimental results of the Hanbury Brown and Twiss method [13] with a different electric time-delay, which were acquired by the synchronous counting between MCP-A and MCP-B. The observation time window was 200 ns, which implies an observation repetition rate of $5 \mathrm{MHz}$. This value was lower than the maximum observation repetition rate $(10 \mathrm{MHz})$ of the time-correlated SP counter used. Therefore, no photon-counting failures had occurred. The total accumulation counting times were $24,000 \mathrm{~s}$ and $12,000 \mathrm{~s}$ [Figs. 4(a) and 4(b), respectively]. These times were limited only by the thermal drift of the fiber probe. The synchronously detected signal was integrated over a time interval of $12.5 \mathrm{~ns}$. In these figures, the synchronous count is zero at the time origin $(\mathrm{t}$ $=0$ ). This clear anti-bunching feature indicates that the coupled QD system was acting as a SP emitter.

Although the synchronous counting was zero at $t=0$, the total counting numbers [143 and 71 in Figs 4(a) and (b), respectively] were smaller than those acquired in previous studies [24]. To confirm the reliability of the present experimental results, discussing the accuracy of 
the SP detection is necessary. The slender bars in Figs. 4(a) and 4(b) represent possible synchronous counts with $90 \%$ significance levels for the total counting number of 143 and 71 , respectively. They were derived by assuming an ideal SP emitter and a binomial probability distribution ". Given these assumptions, the expectation values of the synchronous counts at $t$ $=0$ in Figs. 4(a) and 4(b) were less than 0.27 and 0.54, respectively, with an accuracy of $90 \%{ }^{* *}$. Because the experimental results indicated that the synchronous count was less than 1.0 at $\mathrm{t}=0$, the statistical significances of these values can be estimated to be larger than $99.98 \%$ and $98.64 \%$ for Figs. 4(a) and 4(b), respectively ${ }^{* * *}$. Finally, the plausibilities of SP emission are $99.3 \%$ and $98.6 \%$ for Figs. 4(a) and 4(b), respectively ${ }^{* * *}$. Given this discussion, the SP emitter clearly operated in a reliable manner even under the strong excitation condition $\left(10 \mathrm{~W} / \mathrm{cm}^{2}\right)$. It obtained an internal emission efficiency of 1.0 , which was attributable to the three blockade mechanisms in the system. We are also developing SP emitting devices that operate at room temperature using InAs QDs [8] or $\mathrm{ZnO}$ nanorods with double quantum wells [14]. The results of this research will be published elsewhere.
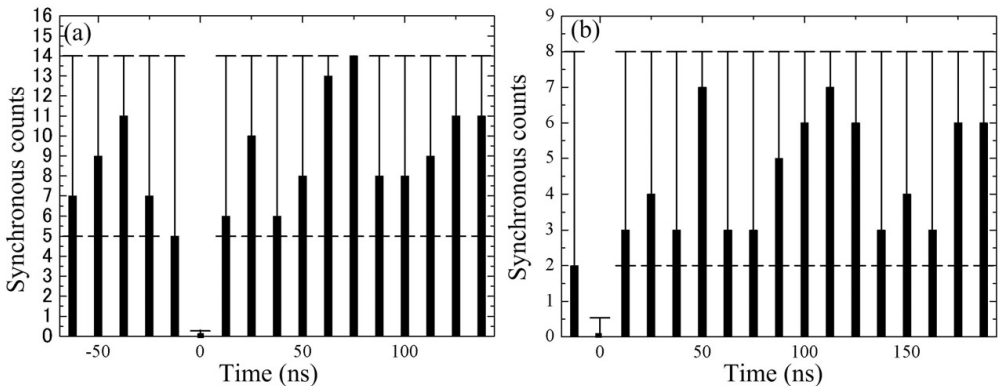

Fig. 4. Experimental results from the Hanbury Brown and Twiss method. The vertical axis represents the numbers of photons that were synchronously counted using MCP-A and MCP-B. The accumulation times of counting were (a) 24,000 s and (b) 12,000 s.

${ }^{*}$ By the binomial probability distribution, the probability of detecting $n$ photons is given by $p_{n}=(1 / 16)^{n}(15 / 16)^{n_{\text {totat }}-n}\left[n_{\text {total }} ! /\left(n_{\text {total }}-n\right) !\right] \cdot(1 / n !)$. Here, $n_{\text {total }}$ is the total counting number of photons, and 16 is the number of thick black bars at $t \neq 0$ in Fig. 4 . Therefore, the probability is $90 \%$ if $5 \leq n \leq 14$, i.e., $\sum_{i=5}^{14} p_{i} \cong 0.9$ in Fig. 4(a). Likewise, the probability is $90 \%$ if $2 \leq n \leq 8$, i.e., $\sum_{i=2}^{8} p_{i} \cong 0.9$ in Fig. 4(b).

${ }^{* *}$ If the probability of detecting $n$ photons at $\mathrm{t}=0$ also follows a binomial distribution, the probability of null synchronous counting is given by $(16 / 17)^{n_{\text {outal }}}$. If $n_{\text {total }}$ is 38 , the probability of

a null synchronous count is $10 \%\left[\cong(16 / 17)^{38}\right]$. Hence, the probability of detecting some

photons at $\mathrm{t}=0$ is $10 \%$, which also means that the deviation from the binomial probability distribution is $90 \%$. In other words, the null synchronous count tells us that a single photon was emitted with an accuracy of $90 \%$. This is because the expectation value of the synchronous count is proportional to $n_{\text {total }}$, irrespective of the probability distribution. The expectation values of the synchronous counts are less than $0.27[=1 \times(38 / 143)]$ at $n_{\text {total }}=143$ and 0.54 [= $1 \times(38 / 71)]$ at $n_{\text {total }}=71$ [Figs. 4(a) and 4(b), respectively] with $90 \%$ accuracy.

${ }^{* * *}$ In the same way as in [15], the accuracy of single-photon emission is estimated as $99.98 \%$ $\left[=1-(16 / 17)^{143}\right]$ and $98.64 \%\left[=1-(16 / 17)^{71}\right]$ at $n_{\text {total }}=143$ and $n_{\text {total }}=71$, respectively.

${ }^{* * * *}$ In the case of a null synchronous count, i.e., a synchronous count of less than 1 , the plausibility of single-photon emission is $99.3 \%$ [ $=1-(1 / 143)]$ and $98.6 \%[=1-(1 / 71)]$ at $n_{\text {total }}=143$ and $n_{\text {total }}=71$, respectively. 


\section{CONCLUDING REMARKS}

In conclusion, we proposed and demonstrated a novel SP emitter using a pair of differently sized QDs coupled by the ONF interaction. SP emission was guaranteed by the three blockade mechanisms: exciton generation, energy transfer, and the emission processes. Through these blockade mechanisms, a SP emission of $99.3 \%$ plausibility with nearly $100 \%$ internal emission efficiency was realized at the operation frequency of $80 \mathrm{MHz}$. Our results were confirmed with $99.98 \%$ accuracy.

\section{References}

[1] E. Knill, R. Laflamme, and G. J. Milburn, "A scheme for efficient quantum computation with linear optics," Nature 409, 46-52 (2001) [doi:10.1038/35051009].

[2] J. I. Cirac, P. Zoller, H. J. Kimble, and H. Mabuchi, "Quantum state transfer and entanglement distribution among distant nodes in a quantum network," Phys. Rev. Lett. 78, 3221-3224 (1997) [doi:10.1103/PhysRevLett.78.3221].

[3] C. Santori, M. Pelton, G. Solomon, Y. Dale, and Y. Yamamoto, "Triggered single photons from a quantum dot," Phys. Rev. Lett. 86, 1502-1505 (2001) [doi:10.1103/PhysRevLett.86.1502].

[4] P. Michler, A. Imamoglu, M. D. Mason, P. J. Carson, G. F. Strouse, and S. K. Buratto, "Quantum correlation among photons from a single quantum dot at room temperature," Nature 406, 968-970 (2000) [doi:10.1038/35023100].

[5] T. Kawazoe, K. Kobayashi, J. Lim, Y. Narita, and M. Ohtsu, "Direct observation of optically forbidden energy transfer between $\mathrm{CuCl}$ quantum cubes via near-field optical spectroscopy," Phys. Rev. Lett. 88, 067404 (2002) [doi:10.1103/PhysRevLett.88.067404].

[6] T. Kawazoe, K. Kobayashi, S. Sangu, and M. Ohtsu, "Demonstration of a nanophotonic switching operation by optical near-field energy transfer," Appl. Phys. Lett. 82, 2957-2959 (2003) [doi:10.1063/1.1571977].

[7] T. Kawazoe, K. Kobayashi, and M. Ohtsu, "Optical nanofountain: A biomimetic device that concentrates optical energy in a nanometric region," Appl. Phys. Lett. 86, 103102 (2005) [doi:10.1063/1.1875734].

[8] T. Kawazoe, K. Kobayashi, K. Akahane, M. Naruse, N. Yamamoto, and M. Ohtsu, "Demonstration of nanophotonic NOT gate using near-field optically coupled quantum dots," Appl. Phys. B 84, 243-246 (2006) [doi:10.1007/s00340-006-2234-x].

[9] N. Sakakura and Y. Masumoto, "Persistent spectral-hole-burning spectroscopy of CuCl quantum cubes," Phys. Rev. B 56, $4051-4055$ (1997) [doi:10.1103/PhysRevB.56.4051].

[10] K. Kobayashi, S. Sangu, T. Kawazoe, and M. Ohtsu, "Exciton dynamics and logic operations in a near-field optically coupled quantum-dot system," J. Luminesc. 112, 117-121 (2005) [doi:10.1016/j.jlumin.2004.09.008].

[11] T. Itoh, Y. Iwabuchi, and M. Kataoka, "Study on the Size and Shape of $\mathrm{CuCl}$ Microcrystals Embedded in Alkali-Chloride Matrices and Their Correlation with Exciton Confinement," phys. Stat. sol. (b) 145, 567-577 (1988) [doi:10.1002/pssb.2221450222].

[12] T. Saiki, S. Mononobe, M. Ohtsu, N. Saito, J. Kusano, "Tailoring a hightransmission fiber probe for photon scanning tunneling microscope," Appl. Phys. Lett. 68, 2612-2614 (1996) [doi:10.1063/1.116198].

[13] R. Hanbury Brown and R. Q. Twiss, "The Question of Correlation between Photons in Coherent Light Rays,” Nature 178, 1447-1448 (1956) [doi:10.1038/1781447a0].

[14] T. Yatsui, S. Sangu, T. Kawazoe, M. Ohtsu, S. J. An, J. Yoo, and G.-C. Yi, "Nanophotonic switch using $\mathrm{ZnO}$ nanorod double-quantum-well structures," Appl. Phys. Lett. 90, 223110 (2007) [doi:10.1063/1.2743949]. 